\title{
Analysis and Simulation of Coherent Antenna
}

Elements and Focuses

A continued research to "Focusing Feasibility in Fractal Array Antennas" [1]

\author{
Prof. Dr. Khalil H. Saydmarie \\ Dept. of Communication Engineering \\ College of Electronic Engineering \\ University of Mosul / Iraq.
}

\author{
Dr. Eanass U. T. Al-Shabkhoon \\ Dept. of Med. Instrumentation Eng. \\ Technical College / Mosul / Iraq \\ Foundation of Technical Education
}

\begin{abstract}
The current research is a result of the study in fractal dimension of antenna applications, but with further extension. The objective of study was to fill the space in near field region by an electromagnetic field at focal point; as a result the space was filled by multiple-pixels known as coherent focuses.

Coherent rays arriving at certain focal point lead to focusing when they are in phase. One of the studied results "Focusing Feasibility in Fractal Array Antennas"; is the relation between the quadratic distances between the elements of focused array and the appearance of coherent focal points. The simulation, analysis, and proof include a deal with the essential mathematical relations which lead to conclude a number of new forms like, definition of focal region, coherent focal region, predication of coherent focal positions.
\end{abstract}

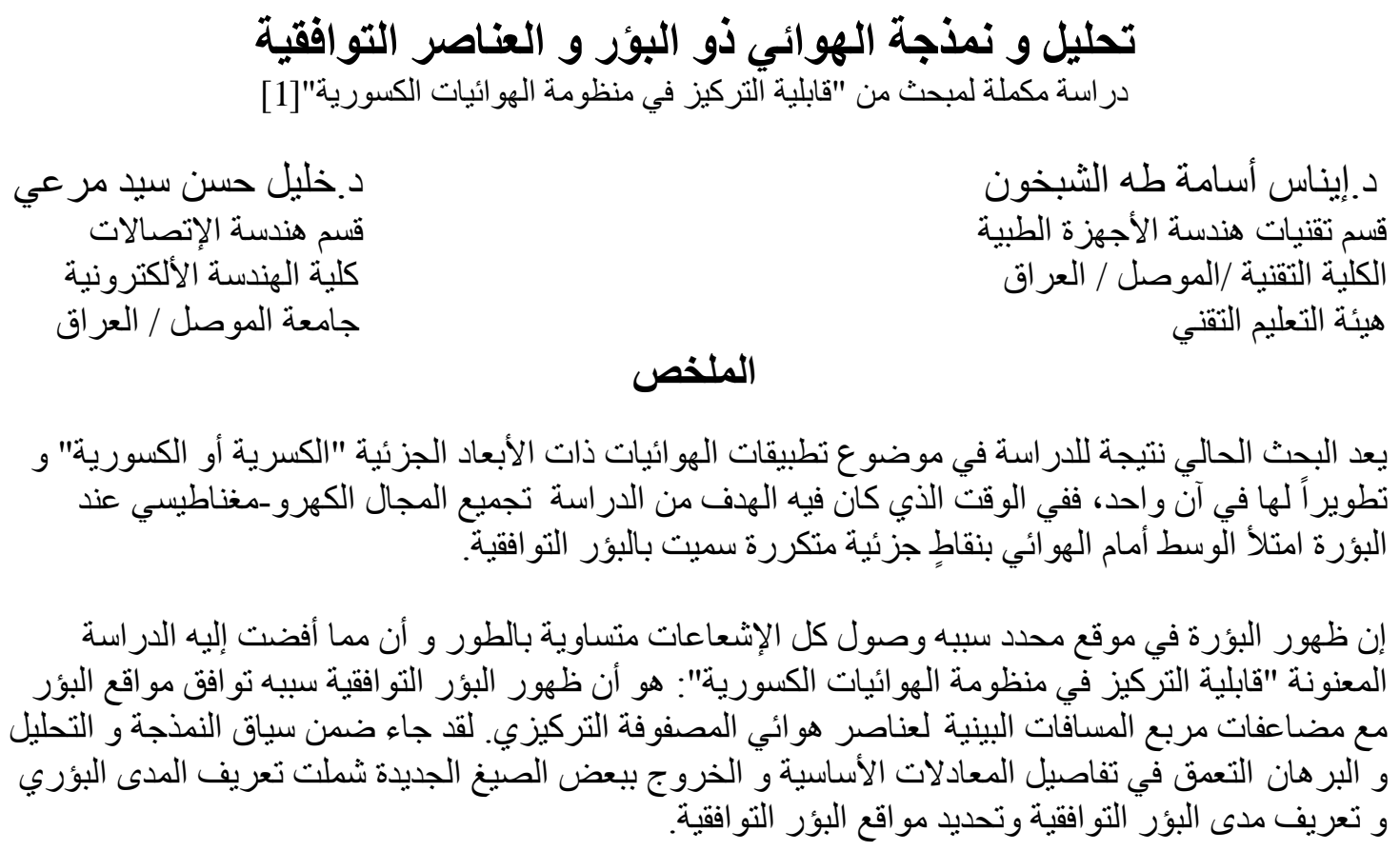




\section{Introduction:}

The radiated electric field from an antenna can be estimated as a function of current distribution across various shapes of its conductive materials. Distribution of electric field is affected by antenna geometry, distance from antenna, antenna material, and the function of excitation signal. The field of point source is used to estimate electric field for an extended antenna or for an array antenna. The solution, that is applied here to describe the variation of electric field at certain distance from a point source, is given in the following equation:

$E=a \frac{\exp [-(\alpha+j \beta) r]}{r}$

and for an N-element array antenna of perpendicular polarization [Steinberg $\left.{ }^{2}\right]$ is:

$E=\sum_{n=1}^{N} a_{n} \frac{\exp \left[-(\alpha+j \beta) r_{n}\right]}{r_{n}}$

where:

E: electric field $[\mathrm{V} / \mathrm{m}]$

$\beta$ : represents phase constant $[\mathrm{rad} / \mathrm{m}]$.

$\alpha$ : represents attenuation constant $[\mathrm{Neper} / \mathrm{m}]$.

$r_{n}$ : represents distance between observation point and isotropic array element $n[m]$.

$\mathrm{a}_{\mathrm{n}}$ : represents a product factor (usually known as element factor), which includes the

effects of excitation, mutual coupling, and others.

Solution like that is acceptable from scientists who are interested in electromagnetic theory since it is compatible with physical truths, where the relation between instantaneous variation of current and gradient field intensity had been found. The intensity of field is attenuated due to surface area of vacuum and due to attenuation factor of medium.

The geometrical optics solution is also applied to solve problem of propagation. Solution is based on ray tracing from source till observation point. Results are affected by previous distances and constants.

Equation (2) is used to describe field variation for two regions:

Near field region

$r<2 L^{2} / \lambda$

Far field region

$r>2 L^{2} / \lambda$

These two regions are defined according to the longest dimension of antenna $\mathbf{L}$. Classification of the two main regions is based on describing the distance $\mathbf{r}$ by a converging series. As a result; polynomial includes linear, quadratic, cubic,......etc phase factors. The higher degree terms are usually neglected which lead to phase error less than $\boldsymbol{\pi / 8}$. So the differences between describing the electric field in near field region and far field region is assuming $\frac{2 \pi r}{\lambda}$ as a linear curve that varies with element position $d_{n}$. Assuming that the variable $\mathbf{r}_{\mathbf{n}}$ have a certain value assigned by $\mathbf{r}_{\mathbf{o}}$, where $\mathbf{r}_{\mathbf{o}}$ represents the distance between the center of antenna layout and the observation point. [Steinberg ${ }^{2}$ ] used another way that differs from [Balanis ${ }^{3}$ ] to define the boundary plane between far field region (Fraunhofer region) and near field region (Fresnel region). The first one takes the value of phase in terms of distance that provides a 
value of one from (Cornu Spiral), then he takes twice the value of distance to define far field region, so he reached at the same result that [Balanis ${ }^{3}$ ] reached.

Notice that [Balanis ${ }^{3}$ ] defined a third region which is known as reactive region which is bounded by $\left(r<0.62 \sqrt{L^{3} / \lambda}\right)$.

In focused arrays, focal point will be observed as long as all radiated rays from various array elements reaching it are in phase [4]. There is an ability of locating focal point in the near region, but it may not be placed toward center of array as it was shown by a large number of simulations [1]. The reason for that will be discussed here in details.

\section{Principle of Study:}

The research is based on the phase conjugate method [4], which locates a virtual isotropic source at focal point, and describes distances to be used for phase compensation as shown in figure (1), in summery there are:

1. Geometrical distance, which is limited by a visible region of isotropic source with respect to array antenna.

2. Approximated distance which is based on resolving the actual distance variation into; first linear and second quadratic "which has the most significant influence in near field region" polynomial terms.

3. Complement distance, which is used to compensate the phase differences that are introduced from the distances differences between focal point and the elements of array antenna.

Now the presented hypothesis can be described as follows:

1. The length of array antenna is within the visible projection of virtual source.

2. The quadratic term of distance cannot exceed the distance from central point of array to the virtual source.

3. The conjugate matching method should be applied, since the assumption deals with virtual isotropic source located at focal point, and providing conjugate phase differences.

The above conditions and assumptions are mentioned to define range of the nearest focal point, where the third condition is applied, while the first and the second ones are competitive conditions used to arrive at the following conclusion (see Appendix):

Focal point cannot be observed for a distance shorter than the half length of array antenna divided by square root of two.

Referring to far focal point that is defined according to [Stienbergs, ${ }^{2}$ ] noting that integration is used to find near field from (Cornu Spiral). That note about fading after the distance $\left(\mathrm{L}^{2} / \lambda\right)$, decay after that distance is extremely steady so it is defined here as a far focal point. Notice that far field region does not mean one must have a point of localization absolutely, it means one will have a focal plane in which electric field is distributed in form of lobes (main, minors, and side lobes). It can be said that far field region follows far focal region, in the other word:

Focal point can be observed at a distance not greater than the quadratic length of antenna divided by two. 
Simulations of many types of linear antenna arrays [1] gave the minimum focal distances shown in Table (1).

Table (1): Nearest Focal Point for One Dimensional Arrays [1] (scale in cm)

\begin{tabular}{|c|c|c|c|c|}
\hline $\begin{array}{c}\text { Antennas } \\
\text { Type }\end{array}$ & $\begin{array}{c}\text { Elements Placement } \\
\text { along x-axis }\end{array}$ & $\begin{array}{c}\text { Phase Excitation } \\
\text { Function }\end{array}$ & $\begin{array}{c}\text { Antenna } \\
\text { Length }\end{array}$ & $\begin{array}{c}\text { Nearest } \\
\text { Focal Point }\end{array}$ \\
\hline Traditional & $\pm[0,2.5,5,7.5,10]$ & Conjugate & 20 & 7 \\
\hline Binary & $\pm[0,2.5,5,7.5,10]$ & Conjugate & 20 & 7 \\
\hline Cantor & $\pm[0,2.5,3.75,5]$ & {$[010111010]$ pi's } & 10 & 3.5 \\
\hline
\end{tabular}

\section{Design of Coherent Antenna (Elements and Focuses):}

The concept of design of coherent antenna is based here on selecting elements positions such that their distances to the focal point differ by an integer multiple of the wavelength. This assumption can be verified by selecting element distances $d_{n}$ at:-

$d_{n}= \pm \sqrt{n^{2} \lambda^{2}+2 n \lambda F_{p}}$

It can also be shown that locating the elements of array at distances of $\left(\mathrm{D}_{\mathrm{n}}=\mathrm{d}_{\mathrm{n}}{ }^{2}\right)$ $D_{n}=n^{2} \lambda^{2}+2 n \lambda F_{p}$

will never violate the above principle of conjugating where the squared values of $d_{n}$ will lead to a multiple of $2 \pi$ complementary phase. So the resulting radiation pattern in near field region of such array looks like far field pattern as one can see in figure (2-1), where color map is used to represent intensity levels of normalized array factor across xy-plane.

Focused antenna array of coherent placement is defined here as:

Focused Coherent Antenna.

Antenna array of coherent placement and focuses is defined here as:

Coherent Antennas Array

The array antenna is designed to have 8-cm length, and four elements which are located at the positions of eq.(3), and a desired focal point located at $1-\mathrm{cm}$ far from the antenna center. Antenna array elements are excited uniformly by a $3 \mathrm{GHz}$ signal to generate far field distribution as in figure (2-1) and as mentioned previously. Bipolar phase excitation is then applied. Array factor (plotted with higher resolution) is found along on-axis as can be seen in figures (2-2), (2-3), and (2-4). Field distribution is found far from $\left(2 \mathrm{~L}^{2} / \lambda\right)$. Field intensity distributes as in figure (2-5) to form coherent focal points. Note that coherent focuses can be observed clearly in far region only. The expected reason of that is the decay factor $(1 / \mathrm{r})$, so its effect is separated and studied alone, while previously the effect of element placement was only discussed by [1]. Coherent focal region is defined in condition that $(1 / \mathrm{r})$ is considered to be studied for acceptable error factor (v). The slope of such curve goes to zero as well as the area $\mathrm{v}$ under the curve is:- 
$\int_{r_{1}}^{r_{2}} \frac{1}{r_{o}} d r_{o} \leq v$

Let $\Delta r=r_{2}-r_{1}$, then the result of integral can be easily found as:

$\left.\Delta r\right|_{v \ll 1}=r_{2}\left(1-e^{-v}\right)$

Field distribution is found for $(\mathrm{v}=0.1)$ and $(\Delta \mathrm{r}=6 \mathrm{~cm})$ to observe coherent focal points as in figure (2-6) in which central focuses are having lowest intensity with respect to side focal points.

v

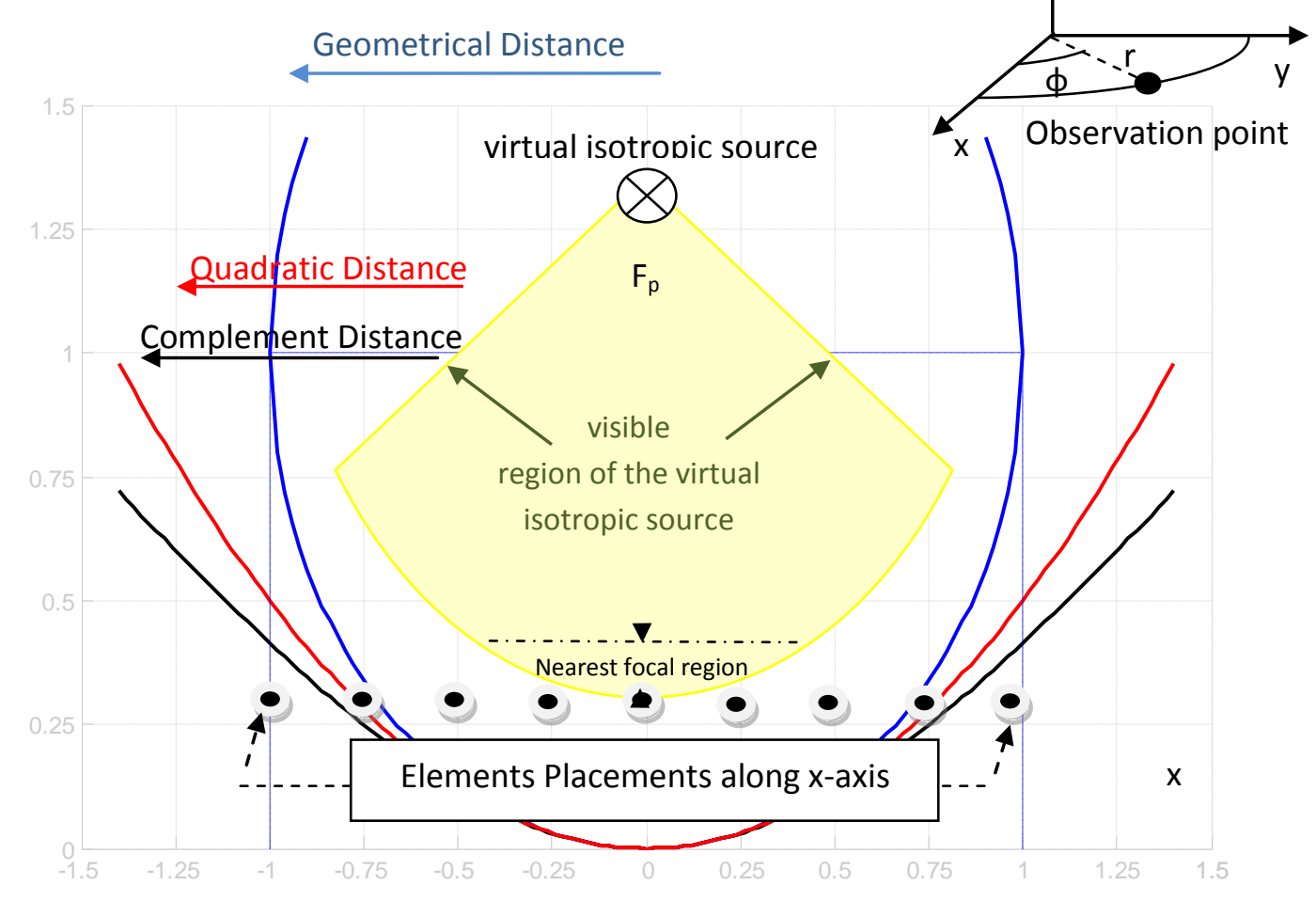

Figure (1): The Description of Array Antenna Layout (scale in cm)

\section{Analysis of Results:}

Analysis is basically dependent on how one looks to the mathematical form of distance variation and how one compares it with standard form. Analysis begins by finding the distance $r_{n}$ from element number $n$ to a general point $p(x, y)$, which can be found in term of element position $\mathrm{D}_{\mathrm{n}}$ as follows:

$r_{n}=\sqrt{\left(x-D_{n}\right)^{2}+y^{2}}$

Let $r_{0}$ refers to distance from origin to point $p\left(x^{2}+y^{2}\right)$, then coherent distance of an element $\mathrm{r}_{\mathrm{cn}}$ can be found in term of element place $\mathrm{D}_{\mathrm{n}}$ as follows:

$r_{c n}=\sqrt{r_{o}^{2}-2 x D_{n}+D_{n}^{2}}$

But if we assume that focal point is at location $\left(\mathrm{F}_{\mathrm{p}}=\lambda, 0\right)$, then:

$\left.D_{n}\right|_{F_{p}=\lambda}=n^{2} \lambda^{2}+2 n \lambda^{2}$

In such case then $D_{n}$ will be multiple of the wavelength;

$\left.D_{n}\right|_{\lambda=\text { int. }}=m \lambda$ 


$$
\begin{aligned}
& \frac{r_{c n}}{\lambda}=\frac{r_{c n}}{\lambda}-m=\frac{r_{c n}}{\lambda}+m \\
& \left.r_{c n}\right|_{r_{o}>\frac{2 L^{2}}{\lambda}}= \pm \sqrt{r_{o}^{2}-2 x D_{n}}
\end{aligned}
$$
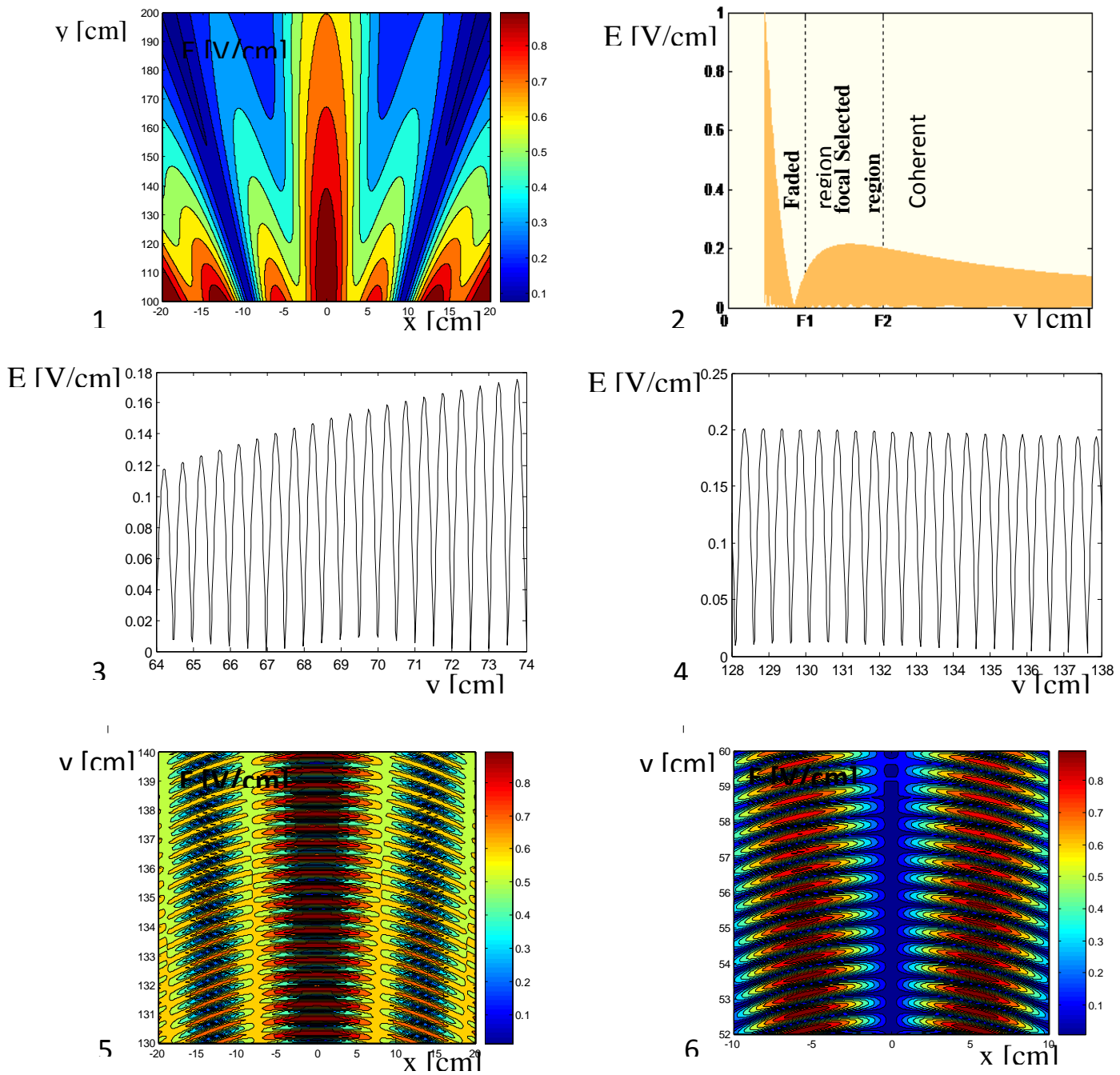

Figure (2): The Normalized Field intensity of antennas array [-8 - $\left.\begin{array}{llll}-3 & 0 & 3 & 8\end{array}\right]$ where;

1.Electric field across xy-plane of uniform excitation function. 2. Electric field along central axis of bipolar excitation function. 3. Zooming central axis of (2) for $\left(\mathrm{y}>\mathrm{F} 1\left\{\mathrm{~L}^{2} / \lambda\right\}\right)$. 4. Zooming central axis of (2) for $\left(y>F 1\left\{2 L^{2} / \lambda\right\}\right)$. 5. Electric field across xy-plane of bipolar excitation function. 6. Electric field of bipolar excitation function for a test cross section $(\Delta \mathrm{r} \approx 6 \mathrm{~cm})$

Final manipulation is considered as mode, where any displacement of phase by a multiple value of $2 \pi$ 's will not affect the absolute value of array factor. Antenna like that has a sinusoidal electric field function and has many focal points separated by half wavelength along on-axis (central-axis). Antenna elements may be considered to be symmetrical about $y$-axis but out of phase, so in absence of central element it can be proven that: 


$$
E=\sum_{n=1}^{N / 2} \frac{\cos \left(\beta \sqrt{r_{o}^{2}-2 x D_{n}}\right)}{r_{n}}
$$

Application of equation (13) cannot be fully successful in near field region while the results in far region are identical to the result of the basic equation (Eq. 2) as can be seen in figure (3) and as can be compared with figure (2-2), where the possibility of minute error is neglected. Error like that will not cause to generate a new curve, but it cause curve distortion as can be seen in figure (4), where a random variable (equal $1 \%$ of wave-length) is added to the electrical length of used rays.

Now let us rewrite the condition of far focal region in term of phase shift $(\theta=\beta r)$, the related phase change of far focal distance is $\pi / 2$, so for all array elements this phase must exceed $\pi / 2$, then critical case can be defined as follows:

$\theta_{c}=\lim _{\lambda / r \rightarrow 4}\left(\frac{2 \pi r}{\lambda}\right)$

Then faded field is observed at certain point. So one should retain again to the definition of distance $r$ in term of polynomial equation to find the region of faded field:

$$
r=r_{o}^{2}-\frac{D_{n} x}{r_{o}}+\frac{D_{n}^{2}}{2 r_{o}}
$$

The third term caused field intensity decrease till zero for any point on central axis. Faded field region is calculated according to:

$$
\frac{\pi D_{N / 2}^{2}}{\lambda r_{o}} \leq \frac{\pi}{2}
$$

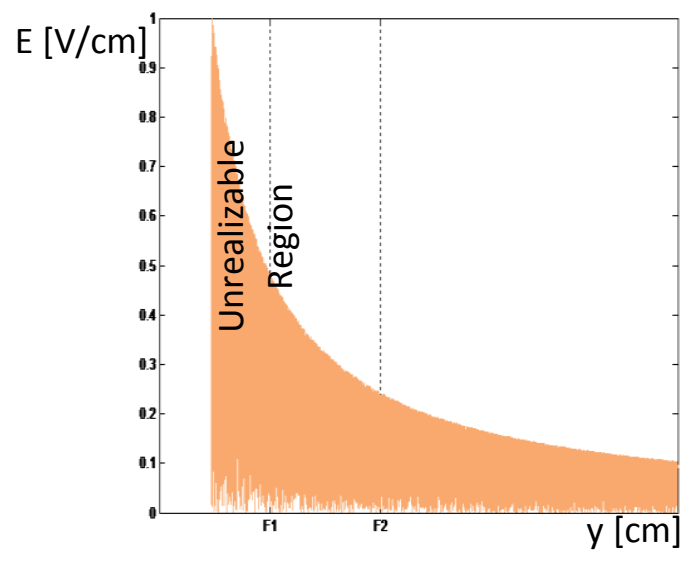

Figure (3): Normalized electric field intensity along the central axis and according to equation (13).

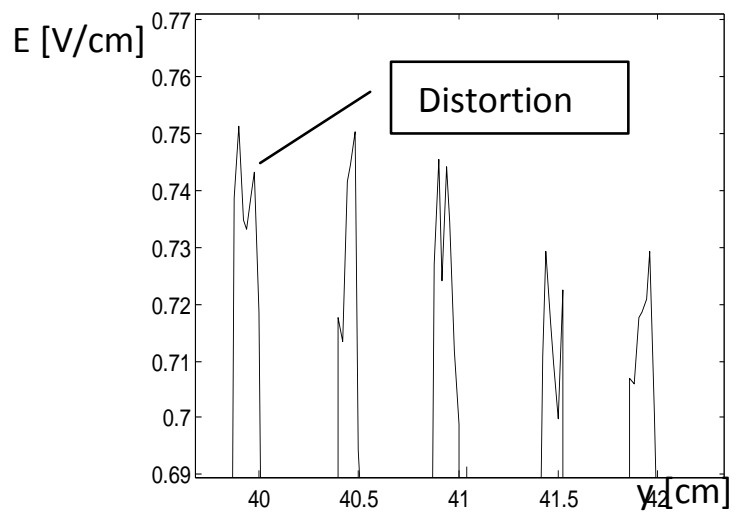

Figure (4): Field distortion due to additive random error in electrical lengths of rays.

The longest $r_{o}$ from which $F_{d}$ is obtained, is calculated for maximum value for $\mathrm{n}$ which is equal to N/2. Faded field region will not be observed by anyway far from $\left(\mathrm{m}^{2} \lambda\right)$ which is equal $64-\mathrm{cm}$ for the array considered here. Referring to the results, it can be found that the faded field region had been displaced towards antenna, where field intensity in near region does not depend on the sinusoidal function only, but it is 
affected by distance attenuation factor (1/r) which also causes the focal point displacement.

\section{Conclusions:}

The importance of these results is not limited by the obtained coherent distribution, but it may be extended to, for example the distribution like that which seems to be uniform distribution if the wavelength is shorter than the studied area. The following notes are also interesting:

The length of desired antenna should be longer than one wavelength. Desired focal point should be placed at a distance equals about one wavelength so that array length is kept reasonable.

1- Field distribution will not be affected so much if a desired focal point is located at a one wavelength or at multiple wavelength distance, but the length of antenna could be much longer.

2- The obtained field distribution may be considered as having coherent or a multifocus according to the scanned area.

3- Classification of distances should be taken into account, where if the influence of $(1 / \mathrm{r})$ factor is neglected by any design procedure in near field, focal point will be displaced.

4- Colored display has been used to show field variation over two dimensional region. The images may differ depending on the used colored scale.

5- The approximate separation between two coherent focuses tends to half wavelength.

6- The approximated form of distances as polynomial seems to be preferable in order to sub-divide the curve of complex equation, then to find a typical solution for each part according to following regions:

a- Selectable focal region:

$r_{2}-\Delta r<r<r_{2}$

b- Expectable faded region:

$\left.\mathrm{F}_{\mathrm{D}}\right|_{\mathrm{Dn}=\mathrm{N} / 2} \leq \mathrm{m}^{2} \lambda$

c- Focal region:

$\frac{\mathrm{L}}{2^{3 / 2}}<r<\frac{\mathrm{L}^{2}}{\lambda}$

d- Coherent focal region:

$$
r>\frac{L^{2}}{\lambda}
$$

\section{Appendix [Proof of nearest focal distance criteria]:}

Virtual distance must equal $F_{p}$ for a point or isotropic source (condition number one in section number two). Quadratic distance is defined by [Stienberg $\left.{ }^{2}\right]$ and it is applied here in term of focal distance as follows:

$$
r_{q}=\frac{x^{2}}{2 F_{p}}
$$

Maximum value of quadratic distance must be given in term of antenna length:

$$
r_{q} \leq \frac{(L / 2)^{2}}{2 F_{p}}
$$


According to condition number two in section number two:

$$
r=F_{p}+\frac{x D_{n}}{F_{p}}-\frac{D_{n}^{2}}{2 F_{p}}+\cdots
$$

Now to compensate distances at the focal position $\left(F_{p}, 0\right)$, the form of complement distance becomes nearest to quadratic form:

$r \cong \frac{D_{n}^{2}}{2 F_{p}}$

and virtual distance $\left(F_{p}\right)$ must be always be greater than complement distance so:

$$
F_{p} \geq \frac{L}{2^{\frac{3}{2}}}
$$

List of Symbols:

\begin{tabular}{|c|c|c|c|}
\hline Symbol & Greek Name & Physical Mean & Unit \\
\hline $\mathrm{a}_{\mathrm{n}}$ & --- & Element factor & $\mathrm{V}$ \\
\hline $\mathrm{d}_{\mathrm{n}}$ & --- & Elements positions ( first case) & $\mathrm{m}$ \\
\hline $\mathrm{D}_{\mathrm{n}}$ & --- & Elements positions (second case) & $\mathrm{m}$ \\
\hline $\mathrm{E}$ & --- & Electric field & $\mathrm{V} / \mathrm{m}$ \\
\hline $\mathrm{F}_{\mathrm{p}}$ & --- & Focal or central distance & $\mathrm{m}$ \\
\hline $\mathrm{L}$ & --- & Antenna Length & $\mathrm{m}$ \\
\hline $\mathrm{m}$ & --- & Mode number & constant \\
\hline $\mathrm{n}$ & --- & Index number of array elements & constant \\
\hline $\mathrm{N}$ & --- & Total number of array elements & constant \\
\hline $\mathrm{r}_{\mathrm{O}}$ & --- & Radius of spherical coordinate & $\mathrm{m}$ \\
\hline$\alpha$ & Alpha & Attenuation constant & Neper/m \\
\hline$\beta$ & Beta & Phase constant & rad./m \\
\hline$\lambda$ & Lambda & Wavelength & $\mathrm{m}$ \\
\hline
\end{tabular}

Acknowledgments: To the thesis ${ }^{[1]}$ discussion committee (2007) for their invaluable suggestions and for those who were interested in the idea of research in technology culture in technical college (2007-2008).

\section{References:}

[1] E. U. T. Al_Shabkhoon, "Feasibility of Focused Fractal Array Antennas", Ph.D. Thesis, College of Engineering, University of Mosul, 2007.

[2] B. D. Stienberg, "Principle of Aperture and Array System Design", John Wiley and Sons, Inc, New York 1972, Chapter 1, Page (3-23).

[3] C. A. Balanis, "Antenna Theory Analysis and Design", Harper and Row, Publisher, Inc., 1982, Chapter 4, Page (100-159).

[4] J. Loane, H. Ling, B. F. Wang, and S.W. Lee, "Experimental Investigation of Retro-Focusing Microwave Hyperthermia Applicator: Conjugate-Field Matching Scheme", IEEE Transaction on Microwave Theory and Techniques, Vol. MTT-34, No.5, May 1986. 